\title{
Flat coordinates of flat Stäckel systems
}

\author{
Krzysztof Marciniak \\ Department of Science and Technology \\ Campus Norrköping, Linköping University \\ 601-74 Norrköping, Sweden \\ krzma@itn.liu.se \\ Maciej Błaszak \\ Faculty of Physics, Division of Mathematical Physics, A. Mickiewicz University \\ Umultowska 85, 61-614 Poznań, Poland \\ blaszakm@amu.edu.pl
}

July 4, 2018

\begin{abstract}
In this article we explicitely construct transformation bewteen separable and flat coordinates for flat Stäckel systems and exploit the structre of these systems in flat coordinates. In the elliptic case these coordinates become well known generalized elliptical coordinates of Jacobi.
\end{abstract}

Keywords and phrases: Hamiltonian systems, completely integrable systems, Stäckel systems, HamiltonJacobi theory, separable potentials

\section{Introduction}

The search for flat coordinates for systems that we a priori know are flat is not easy. This article is devoted to search for flat coordinates for the so called Stäckel systems [1]. Stäckel systems are roughly speaking (for more precise definition, see below) Hamiltonian systems separable in the sense of Hamilton-Jacobi theory by a pointwise transformation to orthogonal coordinates. As such, they are of great importance in theory of classical integrable systems.

In this paper we construct separable flat systems of Stäckel type directly from scratch i.e. from an appropriate separation curve (or an appropriate set of separation relations 2]) and then find flat coordinates for (almost) all flat Stäckel systems of Benenti type. We also establish the signature of metric tensors of these systems. Further, we present the explicit form of many important geometric objects connected to these flat Stäckel systems (namely metric tensors, Killing tensors and separable potentials) in these new coordinates. Thus, we end up with separable flat Hamiltonians written in flat coordinates of respective pseudo-Euclidian metrices.

Our construction encompasses two known cases: Jacobi elliptic coordinates (introduced in [3] and fully described in [4) and Jacobi parabolic coordinates and also one of the less known cases considered recently by Blaszak and Sergyeyev in [5] (but with no degeneration of coordinate systems).

The paper is organized as follows. In Section 2 we remind basic facts about Stäckel systems and in particular about Stäckel systems of Benenti type. In our approach we constructing Stäckel systems directly in their separation coordinates using an appropriate separation relations (separation curve). In Section 3 we present the construction of flat coordinates in the case of real roots in the polynomial that defines a given Benenti system. Section 4 is devoted to expressing various tensor objects in our coordinates and given known and new formulas for a variety of separable potentials. Finally, in Section 5 we consider the case of complex conjugate (but still nondegenerate) roots. The case of degenerated roots is non studied in this paper. 


\section{Stäckel systems}

Consider a set of Darboux coordinates (often called canonical coordinates) $(\lambda, \mu)=\left(\lambda_{1} \ldots,, \lambda_{n}, \mu_{1}, \ldots, \mu_{1}\right)$ on a $2 n$-dimensional Poisson manifold $M$ equipped with a Poisson operator $\Pi$ (so that $\Pi=\sum_{i=1}^{n} \frac{\partial}{\partial \lambda_{i}} \wedge \frac{\partial}{\partial \mu_{i}}$ ). A classical Stäckel system on $M$ is a system of $n$ Hamiltonians (i.e. smooth real-valued functions) $H_{i}$ defined on a dense open subset of $M$ originating from a set of $n$ separation relations [2] of the form:

$$
\sigma\left(\lambda_{i}\right)+\sum_{j=1}^{n} H_{j} \lambda_{i}^{\gamma_{j}}=\frac{1}{2} f\left(\lambda_{i}\right) \mu_{i}^{2}, \quad i=1, \ldots, n,
$$

where $f$ and $\sigma$ are arbitrary functions of one argument and where all $\gamma_{i} \in \mathbf{Z}, i=1, \ldots, n$, and are such that no two $\gamma_{i}$ coincide. Thus, a particular Stäckel system is defined by the choice of integers $\gamma_{1}, \ldots, \gamma_{n}$ and by the choice of functions $f$ and $\sigma$. Customary one can also treat this system of relations as $n$ points on ( $n$ copies of) the following separation curve

$$
\sigma(\lambda)+\sum_{j=1}^{n} H_{j} \lambda^{\gamma_{j}}=\frac{1}{2} f(\lambda) \mu^{2},
$$

in $\lambda \mu$ plane which helps us to avoid writing too many indices. The relations (11) (or $n$ copies of (21) constitute a system of $n$ equations linear in the unknowns $H_{i}$. Solving these relations with respect to $H_{i}$ we obtain $n$ functions $H_{i}=H_{i}(\lambda, \mu)$ on $M$ commuting (since the right-hand sides of formulas (11) commute) with respect to the Poisson operator $\Pi$ :

$$
\left\{H_{i}, H_{j}\right\}_{\Pi} \equiv \Pi\left(d H_{i}, d H_{j}\right)=0 \text { for all } i, j=1, \ldots, n
$$

These functions have the form

$$
H_{i}=\frac{1}{2} \mu^{T} K_{i} G \mu+V_{i}(\lambda) \quad i=1, \ldots, n,
$$

where we denote $\lambda=\left(\lambda_{1}, \ldots, \lambda_{n}\right)^{T}$ and $\mu=\left(\mu_{1}, \ldots, \mu_{n}\right)^{T}$. The functions $H_{i}$ can be interpreted as $n$ quadratic in momenta $\mu$ Hamiltonians on the phase space $M=T^{*} \mathcal{Q}$ cotangent to a Riemannian manifold $\mathcal{Q}$ (so that $\lambda_{1}, \ldots, \lambda_{n}$ are coordinates on $\mathcal{Q}$ ) equipped with the contravariant metric tensor $G$ depending on the function $f$ and the choice of the constants $\gamma_{i}$. They are commonly known as Stäckel Hamiltonians on $M$. Note also that by the very construction of $H_{i}$ the variables $(\lambda, \mu)$ are separation variables for all the Hamiltonians in (3) in the sense that the Hamilton-Jacobi equations associated with the Hamiltonians $H_{i}$ admit a common additively separable solution $W=\sum_{i=1}^{n} W_{i}\left(\lambda_{i}, a\right)$. Further, the objects $K_{i}$ in (3) can be interpreted as (1,1)-type Killing tensors on $\mathcal{Q}$ for the metric $G$. The metric tensor $G$ and all the Killing tensors $K_{i}$ in (3) are diagonal in $\lambda$-variables and it is easy to see that the Killing tensors $K_{i}$ do not depend neither on a particular choice of $f$ nor $\sigma$ : changing $\sigma$ we change the potentials $V_{i}(\lambda)$ while changes of $f$ influence the metric $G$. We define also a (2,0)-type tensors $A_{i}$ (contravariant Killing tensors) by

$$
A_{i}=K_{i} G, \quad i=1 \ldots n
$$

so that since $K_{1}=I$ we have $A_{1}=G$.

A particular subclass of Stäckel systems is given by choosing the separation curve (2) in the form

$$
\sum_{j=1}^{n} H_{j} \lambda^{n-j}=B_{m}(\lambda)\left(\frac{1}{2} \mu^{2}+\lambda^{k}\right), \quad m \in \mathbf{N}, k \in \mathbf{Z}
$$

(so that $f(\lambda)=B_{m}(\lambda)$ while $\sigma(\lambda)=-\lambda^{k} B_{m}(\lambda)$ with an arbitrary fixed integer $k$ ) where

$$
B_{m}(\lambda)=\sum_{j=0}^{m} \lambda^{m-j} \rho_{j}^{(m)}(\beta) \equiv \prod_{j=1}^{m}\left(\lambda-\beta_{j}\right)
$$

is a real polynomial of order $m$ in $\lambda$ with possibly complex roots $\beta_{j}$ (so that $\beta_{j}$ are either real or exist in complex conjugate pairs) that are all assumed to be different (we assume trhoughout the article that 
there is no degeneracy in the roots of the polynomial $\left.B_{m}\right)$. The real coefficients $\rho_{j}^{(m)}(\beta)$ are thus Viète polynomials (signed symmetric polynomials) of the possibly complex constants $\beta_{1}, \ldots, \beta_{m}$ :

$$
\rho_{j}^{(m)}(\beta)=(-1)^{j} \sum_{1 \leq s_{1}<s_{2}<\ldots<s_{j} \leq m} \beta_{s_{1}} \ldots \beta_{s_{j}}, \quad j=1, \ldots, m
$$

and in case of no ambiguity (when $m$ is obvious) we will simply denote them as $\rho_{j}$. The Hamiltonians $H_{i}$ generated by the separation curve (5) constitute a completely integrable system that is called a Stäckel system of Benenti type (or simply a Benenti system) due to S. Benenti's contribution to the study of these objects [6], [7. The Hamiltonians $H_{i}$ have the form (3) with the metric tensor $G$ and the Killing tensors $K_{i}$ given explicitely through

$$
\begin{gathered}
G=\operatorname{diag}\left(\frac{f\left(\lambda_{1}\right)}{\Delta_{1}}, \ldots, \frac{f\left(\lambda_{n}\right)}{\Delta_{n}}\right)=\operatorname{diag}\left(\frac{B_{m}\left(\lambda_{1}\right)}{\Delta_{1}}, \ldots, \frac{B_{m}\left(\lambda_{n}\right)}{\Delta_{n}}\right), \quad \Delta_{i}=\prod_{j \neq i}\left(\lambda_{i}-\lambda_{j}\right) \\
K_{i}=-\operatorname{diag}\left(\frac{\partial q_{i}}{\partial \lambda_{1}}, \cdots, \frac{\partial q_{i}}{\partial \lambda_{n}}\right) \quad i=1, \ldots, n
\end{gathered}
$$

Here and below $q_{i}=q_{i}(\lambda)$ are Viète polynomials in the variables $\lambda_{1}, \ldots, \lambda_{n}$ :

$$
q_{i}(\lambda)=(-1)^{i} \sum_{1 \leq s_{1}<s_{2}<\ldots<s_{i} \leq n} \lambda_{s_{1}} \ldots \lambda_{s_{i}}, \quad i=1, \ldots, n
$$

(cf (6)) that can also be considered as new coordinates on the Riemannian manifold $\mathcal{Q}$ (we will then refer to them as Viète coordinates).

Proposition 1 The metric (7) is flat only for $m \leq n$ and is of constant curvature for $m=n+1$. For higher $m$ it has a non-constant curvature.

One proves this by direct calculation of scalar curvature of (7). The above proposition means that it is meaningful to seek for flat coordinates for Beneti systems only in case when $m=0, \ldots, n$.

Let us now turn our attention to the separable potentials $V_{i}(\lambda)$ in (3) in Benenti case. If we remove the $\lambda^{k}$ term from the right hand side of (5) we receive a geodesic Benenti system (with all potentials in (3) equal to zero). In the non-geodesic case (that is the case generated by the full separation curve (55)) the potentials $V_{i}(\lambda)$ depend on the constants $m$ and $k$ (as well as on the dimension $n$ ) so we will denote them by $V_{i}^{(m, k)}(\lambda)$ or simply by $V_{i}^{(m, k)}$. Notice again that these potentials are generated by the term $\sigma(\lambda)=-\lambda^{k} B_{m}(\lambda)$ in the separation curve (5). Further by $V^{(m, k)}$ we will denote the column vector with components $V_{i}^{(m, k)}$ so that

$$
V^{(m, k)}=\left(V_{1}^{(m, k)}, \ldots, V_{n}^{(m, k)}\right)^{T}
$$

By solving (5) with respect to $H_{i}$ one obtains that

$$
V^{(m, k)}=\sum_{j=0}^{m} \rho_{j}^{(m)}(\beta) U^{(m-j+k)}
$$

where the column vector $U^{(k)}$ represents the so called basic separable potentials related to $\sigma(\lambda)=-\lambda^{k}$ which can be constructed recursively [8, 9 by

$$
U^{(k)}=R^{k} U^{(0)}
$$

with the recursion matrix $R$ of the form

$$
R=\left(\begin{array}{cccc}
-q_{1} & 1 & & \\
-q_{2} & & \ddots & \\
\vdots & & & 1 \\
-q_{n} & 0 & \cdots & 0
\end{array}\right)
$$


and with $U^{(0)}=(0,0, \ldots, 0,1)^{T}$. Note that the formulas (10)-(12) are non tensor in that they are the same in an arbitrary coordinate system, not only in the separation variables $\lambda_{i}$. Note also that for $m=0$ we have $V_{r}^{(0, k)}=U_{r}^{(k)}$ so that for $m=0$ both families of potentials coincide. The potentials $V$ are naturally linear combinations of the basic separable potentials $U$ determined by our specific choice of the function $\sigma(\lambda)$ in (5). This choice is motivated by the fact that the potentials $V$ in flat coordinates generalize the well known potentials as it will be demonstrated below. The "lowest" basic separable potentials have the following form: $U^{(1)}=R U^{(0)}=(0,0, \ldots 0,1,0)^{T}$ up to $U^{(n-1)}=R^{n-1} U^{(0)}=(1,0, \ldots, 0)^{T}$ are trivial (constant), $U^{(n)}=R^{n} U^{(0)}=\left(-q_{1}, \ldots,-q_{n}\right)$ is the first nontrivial positive potential while $U^{(-1)}=R^{-1} U^{(0)}=\left(1 / q_{n}, q_{1} / q_{n}, \ldots, q_{n-1} / q_{n}\right)^{T}$. The "negative" potentials (i.e. potentials obtained for negative $k$ ) are rational functions of $q$ that quickly become complicated with decreasing $k$.

More information on Benenti systems can be found in 10, 11, 12.

\section{Flat coordinates for Stäckel systems - real case}

As we mentioned before, if we restrict ourselves to the case $0 \leq m \leq n$ then the metirc $G$ in (17) is flat so there is a legitimate question of finding flat coordinates for this metric. In this section we construct flat coordinates of $G$ in case where all the roots $\beta_{j}$ of $B_{m}(\lambda)$ are real. So, our aim is to find flat coordinates for the metric tensor $G$ for an arbitrary $m$ between 0 an $n$ and for arbitrary real constants $\beta_{1}, \ldots, \beta_{m}$.

Consider thus the following generating function

$$
\sum_{j=0}^{n-m} z^{n-m-j} a_{j}-\frac{1}{4} \varepsilon \sum_{j=1}^{m} \frac{x_{j}^{2}}{z-\beta_{j}} \equiv \frac{\prod_{j=1}^{n}\left(z-\lambda_{j}\right)}{\prod_{j=1}^{m}\left(z-\beta_{j}\right)}
$$

(where $\varepsilon=+1$ or $\varepsilon=-1$ and where the identity is taken with respect to the variable $z$ ). This function defines (locally) an invertible map between variables $\left(\lambda_{1}, \ldots, \lambda_{n}\right)$ and new variables $\left(x_{1}, \ldots, x_{m}, a_{1}, \ldots, a_{n-m}\right)$ on our manifold $\mathcal{Q}$ whereas the choice of the sign of $\varepsilon$ is governed by the actual sign of the variables in a given region of our Riemannian manifold $\mathcal{Q}$. An easy way to see this is to multiply both sides of (13) by $B_{m}(z) \equiv \prod_{j=1}^{m}\left(z-\beta_{j}\right)$ and compare the coefficients of polynomials on both sides of the equation. We can see that $a_{0}=1$ in the above formula, so in case $m=n$ the generating function (13) attains the form

$$
1-\frac{1}{4} \varepsilon \sum_{j=1}^{n} \frac{x_{j}^{2}}{z-\beta_{j}} \equiv \frac{\prod_{j=1}^{n}\left(z-\lambda_{j}\right)}{\prod_{j=1}^{n}\left(z-\beta_{j}\right)}
$$

which in the regions of the manifold $\mathcal{Q}$ when $\varepsilon<0$ is nothing else as the well known transformation (see [3] and [4]) between the coordinates $\left(x_{1}, \ldots, x_{n}\right)$ and the Jacobi elliptic coordinates $\left(\lambda_{1}, \ldots, \lambda_{n}\right)$. In the case $m=n-1$ the function (13) becomes

$$
z+a_{1}-\frac{1}{4} \varepsilon \sum_{j=1}^{n-1} \frac{x_{j}^{2}}{z-\beta_{j}} \equiv \frac{\prod_{j=1}^{n}\left(z-\lambda_{j}\right)}{\prod_{j=1}^{m}\left(z-\beta_{j}\right)}
$$

which is commonly known as the generating function for transformation between the coordinates $\left(x_{1}, \ldots, x_{n-1}, a_{1}\right)$ and the Jacobi parabolic coordinates $\left(\lambda_{1}, \ldots, \lambda_{n}\right)$. In the case $m=0$ we consider instead of (13) the generating function of the form

$$
\sum_{j=0}^{n} z^{n-j} a_{j} \equiv \prod_{j=1}^{n}\left(z-\lambda_{j}\right)
$$


so that $a_{i}(\lambda)=q_{i}(\lambda)$ i.e. the variables $\left(a_{1}, \ldots, a_{n}\right)$ coincide then with the Viète coordinates (9) while the variables $x_{i}$ are not present at all. One can say that this function is a variant of (13) with both $\varepsilon$ and all $\beta_{i}$ non-present.

Let us now investigate the map between coordinates $\left(\lambda_{1}, \ldots, \lambda_{n}\right)$ and $\left(x_{1}, \ldots, x_{m}, a_{1}, \ldots, a_{n-m}\right)$.

Theorem 2 The map from coordinates $\left(\lambda_{1}, \ldots, \lambda_{n}\right)$ to $\left(x_{1}, \ldots, x_{m}, a_{1}, \ldots, a_{n-m}\right)$ is given by

$$
\begin{gathered}
x_{j}^{2}=-4 \varepsilon \frac{\prod_{k=1}^{n}\left(\beta_{j}-\lambda_{k}\right)}{\prod_{\substack{k=1 \\
k \neq j}}^{m}\left(\beta_{j}-\beta_{k}\right)}, j=1, \ldots m \\
\left(\begin{array}{c}
a_{1} \\
\vdots \\
a_{n-m}
\end{array}\right)=M\left(\begin{array}{c}
q_{1}(\lambda)-\rho_{1}(\beta) \\
\vdots \\
q_{n-m}(\lambda)-\rho_{n-m}(\beta)
\end{array}\right)
\end{gathered}
$$

where $M$ is a square matrix with entries given by

$$
M_{i j}=\left\{\begin{array}{cc}
U_{1}^{(m, m-1+i-j)} & \text { for } j \leq i \\
0 & \text { for } j>i
\end{array} \quad \text { with } i, j=1, \ldots, n-m\right.
$$

where $U_{1}^{(m, m-1+i-j)}$ are basic separable potential given by (11) with the dimension $n$ replaced by $m$.

Proof. To show (15) let us first multiply both sides of (13) by $B_{m}(z)=\prod_{k=1}^{m}\left(z-\beta_{k}\right)$. We receive

$$
B_{m}(z) \sum_{k=0}^{n-m} z^{n-m-k} a_{k}-\frac{1}{4} \varepsilon B_{m}(z) \sum_{k=1}^{m} \frac{x_{k}^{2}}{z-\beta_{k}} \equiv \prod_{k=1}^{n}\left(z-\lambda_{k}\right)
$$

Let us now insert $z=\beta_{j}$ in (18). Since $B_{m}\left(\beta_{j}\right)=0$ we obtain

$$
-\frac{1}{4} \varepsilon x_{j}^{2} \prod_{\substack{k=1 \\ k \neq j}}^{m}\left(\beta_{j}-\beta_{k}\right)=\prod_{k=1}^{n}\left(\beta_{j}-\lambda_{k}\right)
$$

from which (and since $1 / \varepsilon=\varepsilon$ ) we obtain (15). The formula (16) can be obtained by a careful comparison of coefficients of polynomials in (18).

By direct comparison of the coefficients in (18) one can also show that

$$
q_{i}=\sum_{j=0}^{n-m} \rho_{i-j} a_{j}+\frac{1}{4} \varepsilon \sum_{j=1}^{m} \frac{\partial \rho_{i-(n-m)}}{\partial \beta_{j}} x_{j}^{2}, \quad i=1, \ldots, n
$$

which gives us the map from the variables $\left(x_{1}, \ldots, x_{m}, a_{1}, \ldots a_{n-m}\right)$ to the Viète variables (9). In the above formula we use the notation $\rho_{i}=0$ for $i<0$ or for $i>m$ and $\rho_{0}=1$.

Let us now turn to the problem of finding flat coordinates for the metric $G$ generated by (5). Consider the polynomial map (compare with formula (12) in [5])

$$
a_{i}=r_{i}+\frac{1}{4} \sum_{j=1}^{i-1} r_{j} r_{i-j}, \quad i=1, \ldots, n-m
$$

from the variables $\left(r_{1}, \ldots, r_{n-m}\right)$ to $\left(a_{1}, \ldots, a_{n-m}\right)$. This map (20) is injective due to its triangular structure. By theorem of Bialynicki-Birula and Rosenlicht [13] it is then also surjective and therefore bijective and since its Jacobian, as it is easy to see from (20), is equal to 1, by a variant of Jacobian Conjecture (see for example [14] or [15]) the map inverse to (20) is also a polynomial map. Thus, we conclude that 
Proposition 3 The transformation (20) is bijective and its inverse is also a polynomial map.

Combining the maps (15)-(16) and (20) we obtain the map between the variables $\left(\lambda_{1}, \ldots, \lambda_{n}\right)$ and $\left(x_{1}, \ldots, x_{m}, r_{1}, \ldots r_{n-m}\right)$. We are now in position to formulate the main theorem of this paper.

Theorem 4 The metric $G$ defined by (7) attains in coordinates $\left(x_{1}, \ldots, x_{m}, r_{1}, \ldots r_{n-m}\right)$ the form

$$
G=\left(\begin{array}{cc}
\varepsilon I_{m \times m} & 0_{m \times(n-m)} \\
0_{(n-m) \times m} & J_{(n-m) \times(n-m)}
\end{array}\right)
$$

where $I_{k \times k}$ denotes the $k \times k$ identity matrix and $J_{k \times k}$ denotes the $k \times k$ matrix given by $\left(J_{k \times k}\right)_{i j}=\delta_{i, k-j+1}$ i.e. with entries equal to zero everywhere except on the antidiagonal where all the entries are equal to 1. Naturally, $0_{k \times r}$ denotes the $k \times r$ zero matrix.

Proof. One can show this theorem by directly calculating the Jacobian of the map (15)-(16)-(20) but this yields a very tedious calculation. Alternatively, by solving (5) with respect to $H_{i}$ we see that

$$
G=\sum_{k=0}^{m} \rho_{k}^{(m)} G_{k}
$$

(compare with (10). The form of tensors $G_{k}$ in Viète coordinates has been found in [5] so one can easily first transform the tensor $G$ to the Viète coordinates, and then to use the inverse of the Jacobian of the map (19) to transform it to the variables $(x, r)$.

Thus, the variables $\left(x_{1}, \ldots, x_{m}, r_{1}, \ldots r_{n-m}\right)$ are flat but non-orthogonal coordinates for the metric $G$ (while the separation coordinates $\left(\lambda_{1}, \ldots, \lambda_{n}\right)$ are orthogonal but not flat, see (77)). It is now elementary to find the transformation form coordinates $\left(x_{1}, \ldots, x_{m}, r_{1}, \ldots, r_{n-m}\right)$ to the pseudo-Euclidean coordinates for $G$. However, the formulas for Killing tensors and potentials (see below) become much less transparent in these coordinates and this is why we stop at flat coordinates $\left(x_{1}, \ldots, x_{m}, r_{1}, \ldots r_{n-m}\right)$.

Corollary 5 The signature $\left(n_{+}, n_{-}\right)$(where $n_{+}$and $n_{-}$is the number of positive respective negative eigenvalues of $G$ ) of the metric $G$ is (in the real case) given by

$$
\begin{aligned}
& \left(n_{+}, n_{-}\right)=\left(n-\left[\frac{n-m}{2}\right],\left[\frac{n-m}{2}\right]\right) \quad \text { in the region where } \varepsilon=+1 \\
& \left(n_{+}, n_{-}\right)=\left(n-m-\left[\frac{n-m}{2}\right],\left[\frac{n-m}{2}\right]+m\right) \quad \text { in the region where } \varepsilon=-1
\end{aligned}
$$

where $[\alpha]$ denotes the integer part of the number $\alpha$.

This corollary means that the metric $G$ is Euclidean (in the appropriate regions, where $\varepsilon=+1$ ) only in the elliptic case and in the parabolic case (i.e. for $m=n$ and $m=n-1$ ), otherwise it is pseudo-Euclidean. Note also that in case $m=0$ both expressions coincide.

We will now investigate the structure of the Killing tensors $A_{r}$ (defined in (4)) and separable potentials $V$ (defined through formulas (10), (11) and (12)) in the flat coordinates $\left(x_{1}, \ldots, x_{m}, r_{1}, \ldots r_{n-m}\right)$ in the elliptic $(m=n)$ case, in the parabolic $(m=n-1)$ case and in the case of $m=0$ (in the case of arbitrary $m$ the formulas become very complicated and non-transparent). Let us start with the elliptic case $m=n$. The form of the $(2,0)$-type tensors $A_{r}$ in flat coordinates can be calculated by the usual transformation rules for tensors. The result is presented below.

Proposition 6 For $m=n$ the $(2,0)$-tensors $A_{s}(s=1, \ldots, n)$ defined in (4) attain in the flat coordinates $\left(x_{1}, \ldots, x_{n}\right)$ the form

$$
\begin{aligned}
A_{s}^{i j} & =\frac{1}{4} \frac{\partial^{2} \rho_{s}}{\partial \beta_{i} \partial \beta_{j}} x_{i} x_{j}, \quad i \neq j \\
A_{s}^{i i} & =-\varepsilon \frac{\partial \rho_{s}}{\partial \beta_{i}}-\frac{1}{4} \sum_{\substack{k=1 \\
k \neq i}}^{n} \frac{\partial^{2} \rho_{s}}{\partial \beta_{i} \partial \beta_{k}} x_{k}^{2}
\end{aligned}
$$

(no summation over repeated indices is performed here) where $\rho_{s}=\rho_{s}^{(n)}\left(\beta_{1}, \ldots, \beta_{n}\right)$ is given by (6)). 
It is not possible to present the general formula for the potentials $V_{r}^{(n, k)}$ in flat coordinates but we can at least present few potentials with low $k$. We denote $x=\left(x_{1}, \ldots, x_{n}\right)^{T}$ and by $(\cdot, \cdot)$ we denote the usual scalar product in $\mathbf{R}^{n}$. Further, denote

$$
\begin{aligned}
\Gamma_{s} & =-\operatorname{diag}\left(\frac{\partial \rho_{s}^{(n)}}{\partial \beta_{1}}, \cdots, \frac{\partial \rho_{s}^{(n)}}{\partial \beta_{n}}\right) \quad s=1, \ldots, n \\
\Delta & =\operatorname{diag}\left(\beta_{1}, \ldots, \beta_{n}\right) \\
W & =1+\frac{1}{4} \varepsilon\left(x, \Delta^{-1} x\right)
\end{aligned}
$$

(and remember that $\rho_{s}=0$ for $s<0$ and $s>m$ ). In the above notation, we obtain, after some calculations in Maple

$$
\begin{aligned}
V_{s}^{(n, 2)} & =\frac{1}{4} \varepsilon\left(\Gamma_{s} x, \Delta^{2} x\right)+\frac{1}{16}\left(\Gamma_{s} x, x\right)(x, \Delta x)+\frac{1}{64} \varepsilon\left(\Gamma_{s} x, x\right)(x, x)^{2}+\frac{1}{16}(x, x)\left(\Gamma_{s} x, \Delta x\right) \\
V_{s}^{(n, 1)} & =\frac{1}{4} \varepsilon\left(\Gamma_{s} x, \Delta x\right)+\frac{1}{16}\left(\Gamma_{s} x, x\right)(x, x) \\
V_{s}^{(n, 0)} & =\frac{1}{4} \varepsilon\left(\Gamma_{s} x, x\right) \\
V_{s}^{(n,-1)} & =\frac{1}{4} \varepsilon \frac{\left(\Gamma_{s} x, \Delta^{-1} x\right)}{W} \\
V_{s}^{(n,-2)} & =\frac{1}{W^{2}}\left(\frac{1}{4} \varepsilon\left(\Gamma_{s} x, \Delta^{-2} x\right)+\frac{1}{16}\left(\Gamma_{s-1} x, \Delta^{-1} x\right)\left(x, \Delta^{-1} x\right)-\frac{1}{16}\left(\Gamma_{s-1} x, x\right)\left(x, \Delta^{-2} x\right)^{2}\right)
\end{aligned}
$$

For higher positive or negative $k$ these potentials quickly become very complicated. Since $\Gamma_{1}=I$ and $\Gamma_{0}=0$ (due to (6) $)$ we see that

$$
\begin{aligned}
V_{1}^{(n, 2)} & =\frac{1}{4} \varepsilon\left(x, \Delta^{2} x\right)+\frac{1}{8}(x, \Delta x)(x, x)+\frac{1}{64} \varepsilon(x, x)^{3} \\
V_{1}^{(n, 1)} & =\frac{1}{4} \varepsilon(x, \Delta x)+\frac{1}{16}(x, x)^{2} \\
V_{1}^{(n, 0)} & =\frac{1}{4} \varepsilon(x, x) \\
V_{1}^{(n,-1)} & =\frac{1}{4} \varepsilon \frac{\left(x, \Delta^{-1} x\right)}{W} \\
V_{1}^{(n,-2)} & =\frac{1}{4} \varepsilon \frac{\left(x, \Delta^{-2} x\right)}{W^{2}}
\end{aligned}
$$

This family of potentials has been obtained for the first time in [16 (see also [17]). The potential $V_{1}^{(n, 1)}$ is the well known Garnier potential while $V_{1}^{(n, 0)}$ is just harmonic oscillator. Note that both in the Killing tensors $A_{s}$ and in the potentials $V_{s}^{(n, k)}$ the sign $\varepsilon$ is present only at terms with odd powers of $(x, x)$ which is clearly due to (15).

Let us now turn to the parabolic case $m=n-1$. In this case the structure of the Killing tensors $A_{r}$ is more complicated

Proposition 7 For $m=n-1$ the tensors $A_{s}(s=1, \ldots, n)$ attain in the flat coordinates $\left(x_{1}, \ldots, x_{n-1}, r\right)$ 
the form

$$
\begin{aligned}
A_{s}^{i j} & =\frac{1}{4} \frac{\partial^{2} \rho_{s-1}}{\partial \beta_{i} \partial \beta_{j}} x_{i} x_{j}, \quad i \neq j, i, j=1, \ldots, n-1 \\
A_{s}^{i i} & =-\varepsilon \frac{\partial \rho_{s}}{\partial \beta_{i}}-\frac{1}{4} \sum_{\substack{k=1 \\
k \neq i}}^{n-1} \frac{\partial^{2} \rho_{s-1}}{\partial \beta_{i} \partial \beta_{k}} x_{k}^{2}-\varepsilon \frac{\partial \rho_{s-1}}{\partial \beta_{i}} r, \quad i=1, \ldots, n-1 \\
A_{s}^{i n} & =A_{s}^{n i}=\frac{1}{2} \frac{\partial \rho_{s-1}}{\partial \beta_{i}} x_{i}, \quad i=1, \ldots, n-1 \\
A_{s}^{n n} & =\rho_{s-1}
\end{aligned}
$$

(again, with no summation over repeated indices) where $\rho_{s}=\rho_{s}^{(n-1)}\left(\beta_{1}, \ldots, \beta_{n-1}\right)$ is given by (6)).

We will now investigate the potentials $V_{s}^{(n-1, k)}$. Let us slightly change the notation:

$$
\begin{aligned}
\Gamma_{s} & =-\operatorname{diag}\left(\frac{\partial \rho_{s}^{(n-1)}}{\partial \beta_{1}}, \cdots, \frac{\partial \rho_{s}^{(n-1)}}{\partial \beta_{n-1}}\right) \quad s=1, \ldots, n \\
\Delta & =\operatorname{diag}\left(\beta_{1}, \ldots, \beta_{n-1}\right) \\
W & =r+\frac{1}{4} \varepsilon\left(x, \Delta^{-1} x\right)
\end{aligned}
$$

while $(\cdot, \cdot)$ stands now for the standard scalar product in $\mathbf{R}^{n-1}$. We receive, after some calculations, again with the help of Maple

$$
\begin{aligned}
V_{s}^{(n-1,3)} & =-\rho_{s-1} r^{3}+\frac{1}{4} \varepsilon\left(\Gamma_{s-1} x, x\right) r^{2}-\varepsilon\left(\frac{1}{2}\left(\Gamma_{s} x, x\right)-\frac{1}{4} \sum_{j=1}^{n-1} \rho_{s-j-1}\left(x, \Delta^{j} x\right)\right) r \\
& +\frac{1}{4} \varepsilon\left(\Gamma_{s} x, \Delta x\right)+\frac{1}{16}\left(\Gamma_{s-1} x, x\right)(x, x) \\
V_{s}^{(n-1,2)} & =\rho_{s-1} r^{2}-\frac{1}{4} \varepsilon\left(\Gamma_{s-1} x, x\right) r+\frac{1}{4} \varepsilon\left(\Gamma_{s} x, x\right) \\
V_{s}^{(n-1,1)} & =-\rho_{s-1} r+\frac{1}{4} \varepsilon\left(\Gamma_{s-1} x, x\right) \\
V_{s}^{(n-1,0)} & =\rho_{s-1} \\
V_{s}^{(n-1,-1)} & =\frac{1}{W}\left(-\rho_{s-1}+\frac{1}{4} \varepsilon\left(\Gamma_{s-1} x, \Delta^{-1} x\right)\right) \\
V_{s}^{(n-1,-2)} & =\frac{1}{W^{2}}\left(\rho_{s-1}+\frac{1}{4} \varepsilon\left(\Gamma_{s} x, \Delta^{-2} x\right)-\frac{1}{2} \varepsilon\left(\Gamma_{s-1} x, \Delta^{-1} x\right)+\frac{1}{4} \varepsilon\left(\Gamma_{s-1} x, \Delta^{-2} x\right) r\right. \\
& \left.+\frac{1}{16}\left(\Gamma_{s-2} x, \Delta^{-1} x\right)\left(x, \Delta^{-1} x\right)-\frac{1}{16}\left(\Gamma_{s-2} x, x\right)\left(x, \Delta^{-2} x\right)\right)
\end{aligned}
$$

(with $s=1, \ldots, n$ ) and again these formulas become quickly very complicated for higher positive or 
negative $k$. Let us now specify these potentials for case $s=1$. Since $\rho_{0}=1, \Gamma_{1}=I$ while $\Gamma_{0}=0$ we get

$$
\begin{aligned}
V_{1}^{(n-1,4)} & =r^{4}+\frac{3}{4} \varepsilon(x, x) r^{2}-\frac{1}{2} \varepsilon(x, \Delta x) r+\frac{1}{4} \varepsilon\left(x, \Delta^{2} x\right)+\frac{1}{16}(x, x)^{2} \\
V_{1}^{(n-1,3)} & =-r^{3}+\frac{1}{2} \varepsilon(x, x) r+\frac{1}{4} \varepsilon(x, \Delta x) \\
V_{1}^{(n-1,2)} & =r^{2}+\frac{1}{4} \varepsilon(x, x) \\
V_{1}^{(n-1,1)} & =-r \\
V_{s}^{(n-1,0)} & =1 \\
V_{s}^{(n-1,-1)} & =-\frac{1}{W} \\
V_{s}^{(n-1,-2)} & =\frac{1+\frac{1}{4} \varepsilon\left(x, \Delta^{-2} x\right)}{W^{2}}
\end{aligned}
$$

Again, in the above formulas the sign $\varepsilon$ is present only at terms with odd powers of $(x, x)$.

For arbitrary $0 \leq m \leq n-2$ the form of the Killing tensors $A_{s}$ is not so transparent and we will omit it here. Let us however present some results on separable potentials $V^{(m, k)}$ in case $0 \leq m<n-1$. In the case $m=0$ the variables are $\left(r_{1}, \ldots, r_{n}\right)$ and as we mentioned above, $V_{r}^{(0, k)}=U_{r}^{(k)}$ where $U_{r}^{(k)}$ are polynomial (for $k \geq n$ ) or rational (for $k \leq 0$ ) functions of $q_{i}$ given by (11)-(12). Thus (we remember that for $m=0$ we have $q_{i}=a_{i}$, see (14))

$$
V^{(0, k)}(r)=U^{(k)}(r)=\left(\begin{array}{cccc}
-a_{1} & 1 & & \\
-a_{2} & & \ddots & \\
\vdots & & & 1 \\
-a_{n} & 0 & \cdots & 0
\end{array}\right)^{k}\left(\begin{array}{c}
0 \\
\vdots \\
0 \\
1
\end{array}\right), k \in \mathbf{Z}
$$

with

$$
a_{i}=r_{i}+\frac{1}{4} \sum_{j=1}^{i-1} r_{j} r_{i-j}, \quad i=1, \ldots, n
$$

and so the first nontrivial potential is $V^{(0, n)}=\left(-a_{1}, \ldots,-a_{n}\right)^{T}$. The situation is much more complex for the arbitrary $m$ such that $0<m<n-1$. Before we present some results in this generic case, let us introduce a notation similar to used above for the cases $m=n$ and $m=n-1$. We denote

$$
\begin{aligned}
\Gamma_{s} & =-\operatorname{diag}\left(\frac{\partial \rho_{s}^{(m)}}{\partial \beta_{1}}, \cdots, \frac{\partial \rho_{s}^{(m)}}{\partial \beta_{m}}\right) \quad s=1, \ldots, m \\
\Delta & =\operatorname{diag}\left(\beta_{1}, \ldots, \beta_{m}\right)
\end{aligned}
$$

and to shorten the notation we will simply denote $\rho_{s}^{(m)}=\rho_{s}^{(m)}\left(\beta_{1}, \ldots, \beta_{m}\right)$ by $\rho_{s}$. The variables are now $\left(x_{1}, \ldots, x_{m}, r_{1} \ldots, r_{n-m}\right)$ or simply $(x, r)$. This time $(\cdot, \cdot)$ will denote the scalar product in $\mathbf{R}^{n-m}$. Introduce now the column vector of potentials $U^{(k)}=U^{(k)}\left(r_{1}, \ldots r_{n-m}\right), s=1, \ldots, n-m$ given by

$$
U^{(k)}(r)=\left(\begin{array}{cccc}
-a_{1}(r) & 1 & & \\
-a_{2}(r) & & \ddots & \\
\vdots & & & 1 \\
-a_{n-m}(r) & 0 & \cdots & 0
\end{array}\right)^{k}\left(\begin{array}{c}
0 \\
\vdots \\
0 \\
1
\end{array}\right), k \in \mathbf{Z}
$$

with

$$
a_{i}=r_{i}+\frac{1}{4} \sum_{j=1}^{i-1} r_{j} r_{i-j}, \quad i=1, \ldots, n-m
$$


(so that the last trivial potential is $U^{(n-m-1)}=(1, \ldots, 0)^{T}$ ). We obtain, after some Maple calculations

$$
\begin{aligned}
\vdots & \\
V_{s}^{(m, n-m+2)} & =\sum_{j=1}^{n-m} \rho_{s-j} U_{j}^{(n-m+2)}+\frac{1}{4} \varepsilon \sum_{j=0}^{2}\left(\Gamma_{s-(n-m-j)} x, x\right) U_{1}^{(n-m+1-j)} \\
V_{s}^{(m, n-m+1)} & =\sum_{j=1}^{n-m} \rho_{s-j} U_{j}^{(n-m+1)}+\frac{1}{4} \varepsilon \sum_{j=0}^{1}\left(\Gamma_{s-(n-m-j)} x, x\right) U_{1}^{(n-m-j)} \\
V_{s}^{(m, n-m)} & =\sum_{j=1}^{n-m} \rho_{s-j} U_{j}^{(n-m)}+\frac{1}{4} \varepsilon\left(\Gamma_{s-(n-m)} x, x\right) U_{1}^{(n-m-1)} \\
V_{s}^{(m, n-m-1)} & =\rho_{s-1} U_{1}^{(n-m-1)}=\operatorname{const.} \\
\vdots & \\
V_{s}^{(m,-1)} & =\frac{-\sum_{j=1}^{n-m} \rho_{s-j} a_{j-1}+\frac{1}{4} \varepsilon\left(\Gamma_{s-(n-m)} x, \Delta^{-1} x\right)}{a_{n-m}+\frac{1}{4} \varepsilon\left(x, \Delta^{-1} x\right)}
\end{aligned}
$$

Majority of these potentials seem to be new. Potentials higher than $V_{s}^{(m, n-m+2)}$ as well as lower than $V_{s}^{(m,-1)}$ contain terms at least quadratic in $(x, x)$ and are too complicated to present it here.

\section{Flat coordinates for Stäckel systems in the complex case}

We will now investigate the case of complex conjugate roots in the polynomial $B_{m}(\lambda)$. Assume thus that the first $2 p(2 p \leq m)$ roots $\beta_{j}$ in $B_{m}(\lambda)$ are pairwise complex conjugate with nonzero imaginary parts:

$$
\overline{\beta_{2 r-1}}=\beta_{2 r}, r=1, \ldots, p, \operatorname{Im}\left(\beta_{r}\right) \neq 0
$$

It is easy to check that $x_{j}^{2}$ given by (15) are then pairwise complex conjugate as well: $\overline{x_{2 r-1}}= \pm x_{2 r}$, $r=1, \ldots, p$ although the generating function (13)

$$
\sum_{j=0}^{n-m} z^{n-m-j} a_{j}-\frac{1}{4} \varepsilon \sum_{j=1}^{m} \frac{x_{j}^{2}}{z-\beta_{j}} \equiv \frac{\prod_{j=1}^{n}\left(z-\lambda_{j}\right)}{\prod_{j=1}^{m}\left(z-\beta_{j}\right)}
$$

remains real as $B_{m}(\lambda)=\prod_{j=1}^{m}\left(z-\beta_{j}\right)$ is real and since

$$
\frac{x_{2 r-1}^{2}}{z-\beta_{2 r-1}}+\frac{x_{2 r}^{2}}{z-\beta_{2 r}}
$$

is real for any $r=1, \ldots, p$. Let us now define new real variables

$$
\eta_{2 s-1}=\frac{x_{2 s-1}+x_{2 s}}{\sqrt{2}}, \eta_{2 s}=\frac{x_{2 s-1}-x_{2 s}}{\sqrt{2} i}, \quad s=1, \ldots, p
$$

The transformation inverse to (21) is

$$
x_{2 s-1}=\frac{1}{\sqrt{2}}\left(\eta_{2 s-1}+i \eta_{2 s}\right), x_{2 s}=\frac{1}{\sqrt{2}}\left(\eta_{2 s-1}-i \eta_{2 s}\right), \quad s=1, \ldots, p
$$

We are now in position to formulate a theorem analogous to Theorem 4 . 
Theorem 8 The metric $G$ defined by (7) attains in coordinates $(\eta, x, r)=\left(\eta_{1}, \ldots, \eta_{2 p}, x_{2 p+1}, \ldots, x_{m}, r_{1}, \ldots r_{n-m}\right)$ the form

$$
G=\left(\begin{array}{ccc}
\varepsilon D_{2 p \times 2 p} & 0 & 0 \\
0 & \varepsilon I_{(m-2 p) \times(m-2 p)} & 0 \\
0 & 0 & J_{(n-m) \times(n-m)}
\end{array}\right)
$$

where $D_{2 p \times 2 p}$ is a $2 p \times 2 p$ diagonal matrix with intertwined entries 1 and -1 :

$$
D_{2 p \times 2 p}=\operatorname{diag}(1,-1, \ldots, 1,-1)
$$

and where as before $I_{k \times k}$ denotes the $k \times k$ indetity matrix and $J_{k \times k}$ denotes the $k \times k$ matrix given by $\left(J_{k \times k}\right)_{i j}=\delta_{i, k-j+1}$ i.e. with entries equal to zero everywhere except on the antidiagonal where all the entries are equal to 1 . As before, the symbol 0 in the above formula denotes a zero matrix of appropriate dimensions.

Proof. One can prove this theorem similarly as one proves Theorem 4 As before, we observe that

$$
G=\sum_{k=0}^{m} \rho_{k}^{(m)} G_{k}
$$

(with the real coefficients $\rho_{k}^{(m)}$ ). The form of tensors $G_{k}$ in Viète coordinates is known [5] so one can easily first transform the tensor $G$ to the Viète coordinates, and then use the inverse of the Jacobian of the map (19) to transform it to the variables $(\eta, x, r)$.

Note that the map (19)

$$
q_{i}=\sum_{j=0}^{n-m} \rho_{i-j} a_{j}+\frac{1}{4} \varepsilon \sum_{j=1}^{m} \frac{\partial \rho_{i-(n-m)}}{\partial \beta_{j}} x_{j}^{2}, \quad i=1, \ldots, n
$$

is actually a real map even in the complex case. The formal (algebraic, not complex-analytic) derivatives of $\rho_{i-(n-m)}$ with respect to those of $\beta_{j}$ that are complex, together with the corresponding complex $x_{j}^{2}$, enter the second sum in (22) in complex conjugate pairs, so that this sum is indeed real.

Thus, the variables $(\eta, x, r)=\left(\eta_{1}, \ldots, \eta_{2 p}, x_{2 p+1}, \ldots, x_{m}, r_{1}, \ldots r_{n-m}\right)$ are flat but not orthogonal while the original variables $\left(\lambda_{1}, \ldots, \lambda_{n}\right)$ are orthogonal but not flat.

Corollary 9 The signature $\left(n_{+}, n_{-}\right)$of the metric $G$ is in the complex case given by

$$
\begin{aligned}
& \left(n_{+}, n_{-}\right)=\left(n-\left[\frac{n-m}{2}\right]-p,\left[\frac{n-m}{2}\right]+p\right) \quad \text { in the region where } \varepsilon=+1 \\
& \left(n_{+}, n_{-}\right)=\left(n-m-\left[\frac{n-m}{2}\right]+p,\left[\frac{n-m}{2}\right]+m-p\right) \quad \text { in the region where } \varepsilon=-1
\end{aligned}
$$

where $[\alpha]$ denotes the integer part of the number $\alpha$.

It is not possible to write down Killing tensors in the complex case in any reasonably compact form even for the elliptic case, the subcases become too many, albeit it is not difficult to calculate these tensors for any given choice of parameters $n, m, p$. There is on the other hand no change to the potentials $V_{s}^{(m, k)}$ in the complex case as the function $\sigma(\lambda)=-\lambda^{k} B_{m}(\lambda)$ in (5) is real even in the complex case. Of course the explicit form of the potentials do change. For example, in the complex elliptic case, in the variables $(\eta, x, r)=\left(\eta_{1}, \ldots, \eta_{2 p}, x_{2 p+1}, \ldots, x_{n}\right)$, the potentials $V_{s}^{(n, k)}$, given in the previous section, do change its explicit form. The scalar product $(x, x)$ (which is real due to the fact that now $\overline{x_{2 r-1}}= \pm x_{2 r}$, $r=1, \ldots, p)$ is given explicitly as

$$
(x, x)=\sum_{s=1}^{p}\left(\eta_{2 s-1}^{2}-\eta_{2 s}^{2}\right)+\sum_{s=2 p+1}^{n} \eta_{s}^{2}
$$


and so on. The fact that $V_{s}^{(n, k)}$ are real also in the complex case can be easily seen, as all the expressions $(x, x),\left(x, \Delta^{p} x\right)$ for $p \in \mathbf{Z},\left(\Gamma_{s} x, x\right)$ and $\left(\Gamma_{s} x, \Delta^{p} x\right)$ are real. For example, $\left(\Gamma_{s} x, x\right)$ contains apart from the real terms also pairs of complex terms of the form

$$
-\frac{\partial \rho_{s}^{(n)}}{\partial \beta_{2 i-1}} x_{2 i-1}^{2}-\frac{\partial \rho_{s}^{(n)}}{\partial \beta_{2 i}} x_{2 i}^{2}
$$

(where again we have formal i.e. non-complex algebraic derivatives). We easily see that each such pair and so the whole expression $\left(\Gamma_{s} x, x\right)$ is real.

\section{Conclusions}

In this article we presented flat coordinates for majority of flat Stäckel systems. These coordinates coincide in the elliptic (i.e. when $m=n)$ and in the parabolic $(m=n-1)$ cases with the well known generalized Jacobi elliptic (respectively parabolic) coordinates. The only Stäckel systems that have not been covered by our construction are those that are generated by the separation curve (5D) in the case when some of the roots of the polynomial $B_{m}(\lambda)$ has algebraic multiplicity larger than 1 . On the other hand, in [5] the authors considered the completely degenerated case i.e. when all $\beta_{i}$ coincide (and are equal to zero). For the case $m=0$ our formulas coincide with the formulas obtained there.

In this paper we also presented - in the elliptic $m=n$ and parabolic $m=n-1$ case - a compact form of Killing tensors of Stäckel systems in these flat coordinates and also a compact form of their separable

potentials $V_{s}^{(m, k)}$. They contain the well known Garnier system and they encompass for example both families of separable potentials found in [16]. We also presented the form of the first few potentials $V_{s}^{(m, k)}$ in the case $m=0$ i.e. the formulas for $V^{(0, k)}(r)=U^{(k)}(r)$ in flat coordinates.

\section{Acknowledgement}

Both authors were partially supported by The Royal Swedish Academy of Sciences grant no FOA13Magn088 .

\section{References}

[1] P. Stäckel, Habilitationsschrift, Halle 1891

[2] Sklyanin, E. K. Separation of variables - new trends. Progr. Theoret. Phys. Suppl. 118, (1995), $35-60$.

[3] C.G. Jacobi, J. Reine Angew. Math., Bd XIX,

[4] C. G. Jacobi, Vörlesungen über Dynamik, 9, 26-29, Georg Reimer, Berlin 1866

[5] Błaszak, M; Sergyeyev, A., Natural coordinates for a class of Benenti systems. Phys. Lett. A 365 (2007), no. 1-2, 28-33.

[6] Benenti, S., Inertia tensors and Stäckel systems in the Euclidean spaces. Rend. Sem. Mat. Univ. Politec. Torino 50 (1992), no. 4, 315-341 (1993).

[7] Benenti, S., Intrinsic characterization of the variable separation in the Hamilton-Jacobi equation. J. Math. Phys. 38 (1997), no. 12, 6578-6602.

[8] Błaszak, M; Sergyeyev, A., Generalized Stäckel systems. Phys. Lett. A 375 (2011), no. 27, 2617-2623.

[9] Błaszak, M.; Marciniak, K., On reciprocal equivalence of Stäckel systems, Studies in Appl. Math. 129 (2012) 26-50. 
[10] Błaszak, M., Separable systems with quadratic in momenta first integrals, J. Phys. A 38 (2005) $1667-1685$.

[11] Blaszak, M. ; Marciniak, K., Stäckel systems generating coupled KdV hierarchies and their finite-gap and rational solutions, J. Phys. A 41 (2008), no. 48, 485202

[12] K. Marciniak, M. Blaszak, Construction of coupled Harry Dym hierarchy and its solutions from Stäckel systems, Nonlinear Analysis: Theory, Methods and Applications 73 (2010) 3004-3017, http://dx.doi.org/10.1016/j.na.2010.06.067

[13] Białynicki-Birula, A. and Rosenlicht, M. Injective morphisms of real algebraic varieties. Proc. Amer. Math. Soc. 131962 200-203.

[14] Kurdyka, K. and Rusek, K. Polynomial-rational bijections of $\mathbf{R}^{n}$. Proc. Amer. Math. Soc. 102 (1988), no. 4, 804-808.

[15] Bass, H., Connell, E. H. and Wright, D. The Jacobian conjecture: reduction of degree and formal expansion of the inverse. Bull. Amer. Math. Soc. (N.S.) 7 (1982), no. 2, 287-330

[16] Wojciechowski, S. Review of the recent results on integrability of natural Hamiltonian systems. Systèmes dynamiques non linéaires: intégrabilité et comportement qualitatif, 294-327, Sém. Math. Sup., 102, Presses Univ. Montréal, Montreal, QC, 1986.

[17] Antonowicz, M.; Rauch-Wojciechowski S., How to construct finite dimensional bi-Hamiltonian systems from soliton equations: Jacobi integrable potentials, J. Math. Phys. 33 (1992) 2115 